\title{
EMBEDDINGS OF LOCAL GENERALIZED MORREY SPACES BETWEEN WEIGHTED LEBESGUE SPACES
}

\author{
ALEXANDRE ALMEIDA* AND STEFAN SAMKO
}

\begin{abstract}
We prove that local generalized Morrey spaces are closely embedded between weighted Lebesgue spaces. We show that such embeddings are strict in all the cases under consideration by constructing counterexamples. As a consequence, continuous embeddings between generalized Morrey spaces and generalized Stummel spaces are established, as well as between Stummel classes (vanishing Stummel spaces). In particular, we obtain embeddings into a new Stummel class of functions with some vanishing property at infinity. We also partially improve a known result on the coincidence of Stummel spaces with a modification of Morrey spaces where the supremum norm is replaced by an integral $L^{p}$-norm.
\end{abstract}

\section{INTRODUCTION}

Morrey spaces are widely used in applications to regularity properties of solutions to PDE including the study of Navier-Stokes equations. We only refer to the books $[1,5,14,21,22]$ and the overview [15] for additional references and basic properties and generalizations of Morrey spaces. An important feature of these function spaces is that they allow to describe local properties of functions better than Lebesgue spaces.

Since Morrey spaces on $\mathbb{R}^{n}$ are not embedded into Lebesgue spaces, it was always of interest to find embeddings of such spaces into weighted Lebesgue spaces. Up to authors' knowledge, the first such a result appears in [10, p. 132] who showed that global classical Morrey spaces $L^{p, \lambda}\left(\mathbb{R}^{n}\right)$ are embedded into the weighted Lebesgue space $L^{p}\left(\mathbb{R}^{n}, w\right)$ with the weight $w(x)=(1+|x|)^{-\mu}, \mu>\lambda$. As a close result, we mention also embeddings of inhomogeneous classical Morrey spaces into power weighted Lebesgue spaces given in [17, Theorem 3.1] (see also [23, Theorem 2.8]). The results in [10] and [17] were recently extended in [2, Section 6$]$ to the case of locally bounded weights satisfying $\int_{0}^{\infty} w(t) t^{\lambda-1} d t<\infty$. It is also of special interest to have embeddings of local Morrey spaces, which are larger than the global ones, into weighted spaces. In the case of bounded domains in $\mathbb{R}^{n}$ such embeddings were obtained in [18, Theorem 2.1] for local generalized Morrey spaces $L_{\left\{x_{0}\right\}}^{p, \varphi}(\Omega)$ with weights $w(x)=\frac{1}{\varphi\left(\left|x-x_{0}\right|\right) \ln ^{1+\varepsilon} \frac{A}{\left|x-x_{0}\right|}}, \varepsilon>0, A>\operatorname{diam} \Omega$.

Date: August 23, 2017.

2010 Mathematics Subject Classification. 46E30, $42 \mathrm{~B} 35$.

Key words and phrases. Local Morrey space, global Morrey space, generalized Morrey space, weighted Lebesgue space, embeddings, Stummel space, Stummel classes.

* Corresponding author.

This work was supported in part by the Portuguese Foundation for Science and Technology (FCT Fundação para a Ciência e a Tecnologia), through CIDMA - Center for Research and Development in Mathematics and Applications, within project UID/MAT/04106/2013. The second author was supported by the Russian Foundation for Basic Research under grant 15-01-02732. 
Our main goal is to shed more light on this problem of embedding by proving the bilateral embeddings

$$
L^{p}\left(\mathbb{R}^{n}, \frac{1}{\varphi_{x_{0}}}\right) \hookrightarrow L_{\left\{x_{0}\right\}}^{p, \varphi}\left(\mathbb{R}^{n}\right) \hookrightarrow L^{p}\left(\mathbb{R}^{n}, \frac{w_{x_{0}}}{\varphi_{x_{0}}}\right)
$$

with the notation (2.2) and (3.1). As regards the weight $w$, we assume that it is almost increasing on $\left(0, r_{0}\right)$ and almost decreasing on $\left(r_{0}, \infty\right)$, for some $r_{0}>0$, and it satisfies the integral condition $\int_{0}^{\infty} \frac{w(t)}{t} d t<\infty$. The left-hand side embedding is strict for any increasing function $\varphi$. We show that the right-hand side embedding is also strict under some additional assumption on $w / \varphi$.

As an immediate consequence of our main result, we obtain theorems on embedding between global generalized Morrey spaces and Stummel or vanishing Stummel spaces. This generalizes embeddings between Morrey and Stummel spaces given in [18] and reproves in a different form embeddings obtained in [7]. In comparison with [7], we treat the so-called Stummel class rather as a closed subspace of Stummel space than just a set of functions with Stummel property. This vanishing property plays an important role in some applications to PDE, see for instance [4], [11, 12], [16] and [25]. We also consider a new notion of Stummel property related to behavior of functions at infinity, see Definition 2.2 and Corollary 4.2.

We also partially improve a known result on coincidence of generalized Stummel spaces with a modified global Morrey spaces where the supremum norm on $r$ is replaced by an integral $L^{p}$-(quasi)norm.

In the recent paper [8] embedding results were obtained within the scale of weighted Morrey spaces. In a sense, the approach followed in [8] is "dual" since there the weights are considered from the source space side.

The paper is organized as follows. After some notation and preliminaries on generalized Morrey spaces and generalized Stummel spaces, in Section 3 we prove the above mentioned bilateral embeddings of local generalized Morrey spaces between two weighted Lebesgue spaces, and show the strictness of these embeddings. In Section 4 we apply the previous results to obtain embeddings of global generalized Morrey spaces between generalized Stummel spaces. Finally, in Section 5, we deal with an equivalent (quasi)norm for generalized Stummel spaces.

\section{PRELIMINARIES}

We denote by $B(x, r)$ the open ball in $\mathbb{R}^{n}$ centered at $x \in \mathbb{R}^{n}$ with radius $r>0$. If $E \subseteq \mathbb{R}^{n}$ is a measurable set, then $|E|$ stands for its (Lebesgue) measure and $\chi_{E}$ denotes its characteristic function. The notation $X \hookrightarrow Y$ stands for continuous embeddings from the (quasi)normed space $X$ into the (quasi)normed space $Y$. We use $c$ as a generic positive constant, i.e., a constant whose value may change with each appearance. The expression $f \lesssim g$ means that $f \leq c g$ for some independent constant $c$, and $f \approx g$ means $f \lesssim g \lesssim f$.

A function $\phi: \mathbb{R}_{+} \rightarrow \mathbb{R}_{+}$is called almost increasing if $\sup _{t<s} \frac{\phi(t)}{\phi(s)}<\infty$. A function $\phi$ is called almost decreasing if $1 / \phi$ is almost increasing.

As usual, $L^{p}\left(\mathbb{R}^{n}\right)$ denotes the standard Lebesgue space (quasi)normed by

$$
\|f\|_{L^{p}\left(\mathbb{R}^{n}\right)}=\left(\int_{\mathbb{R}^{n}}|f(x)|^{p} d x\right)^{1 / p}, \quad 0<p<\infty .
$$


For a weight $w$ (i.e., a positive measurable function on $\mathbb{R}^{n}$ ), the classical weighted Lebesgue space $L^{p}\left(\mathbb{R}^{n}, w\right)$ is (quasi)normed by

$$
\|f\|_{L^{p}\left(\mathbb{R}^{n}, w\right)}=\left(\int_{\mathbb{R}^{n}}|f(x)|^{p} w(x) d x\right)^{1 / p}, \quad 0<p<\infty .
$$

2.1. Generalized Morrey spaces. Let $0<p<\infty$ and let $\varphi:(0, \infty) \rightarrow(0, \infty)$ be a measurable function. The generalized (homogeneous) global Morrey space $L^{p, \varphi}\left(\mathbb{R}^{n}\right)$ consists of all locally $p$-integrable functions $f$ on $\mathbb{R}^{n}$ such that

$$
\|f\|_{L^{p, \varphi}\left(\mathbb{R}^{n}\right)}:=\sup _{x \in \mathbb{R}^{n}, r>0}\left(\frac{1}{\varphi(r)} \int_{B(x, r)}|f(y)|^{p} d y\right)^{1 / p}<\infty .
$$

The functional (2.1) is a (quasi)norm in $L^{p, \varphi}\left(\mathbb{R}^{n}\right)$. Notice that the classical Morrey space corresponds to the choice $\varphi(r)=r^{\lambda}$, with $0 \leq \lambda \leq n$, and it is often denoted by $L^{p, \lambda}\left(\mathbb{R}^{n}\right)$. In particular $L^{p, 0}\left(\mathbb{R}^{n}\right)=L^{p}\left(\mathbb{R}^{n}\right)$ is the classical Lebesgue space.

We can also consider localized versions of these spaces by fixing the point where the local integrability properties of the functions are studied. More precisely, for a fixed point $x_{0} \in \mathbb{R}^{n}$, the local generalized Morrey space $L_{\left\{x_{0}\right\}}^{p, \varphi}\left(\mathbb{R}^{n}\right)$ is the space of all locally $p$-integrable functions $f$ on $\mathbb{R}^{n}$ equipped with the (quasi)norm

$$
\|f\|_{L_{\left\{x_{0}\right\}}^{p, \varphi}\left(\mathbb{R}^{n}\right)}:=\sup _{r>0}\left(\frac{1}{\varphi(r)} \int_{B\left(x_{0}, r\right)}|f(y)|^{p} d y\right)^{1 / p} .
$$

Of course we have $L^{p, \varphi}\left(\mathbb{R}^{n}\right) \hookrightarrow L_{\left\{x_{0}\right\}}^{p, \varphi}\left(\mathbb{R}^{n}\right)$ for every point $x_{0} \in \mathbb{R}^{n}$.

The interest in studying generalized Morrey spaces comes not only from theoretical reasons, but also from their important role in getting optimal Sobolev type embeddings for critical exponents (see, for instance, [3] and [24]).

Everywhere below we shall assume that the function $\varphi$ is measurable and increasing. In general, to deal with the global Morrey spaces, it is usually assumed that, in addition, $\varphi(t) / t^{n}$ is decreasing. When we work with the localized version of Morrey spaces we do not assume the last assumption to hold. In fact, we always have $L_{\left\{x_{0}\right\}}^{p, \varphi}\left(\mathbb{R}^{n}\right) \neq\{0\}$, while the global space may be trivial without the latter additional assumption.

The next lemma shows that global generalized Morrey spaces on $\mathbb{R}^{n}$ in general are not embedded into Lebesgue spaces.

Lemma 2.1. Let $0<p<\infty$ and $\varphi$ be increasing. If

$$
\frac{\varphi(t)}{t^{\varepsilon}} \text { is almost increasing }
$$

for some $\varepsilon>0$, and

$$
\frac{\varphi(t)}{t^{n}} \text { is almost decreasing, }
$$

then the function

$$
f_{0}(x):=\left(\frac{\varphi\left(\left|x-x_{0}\right|\right)}{\left|x-x_{0}\right|^{n}}\right)^{1 / p}, \quad x_{0} \in \mathbb{R}^{n},
$$

belongs to $L^{p, \varphi}\left(\mathbb{R}^{n}\right)$ but it does not belong to $L^{p}\left(\mathbb{R}^{n}\right)$. 
Proof. We take $x_{0}=0$ for simplicity. If $|x|<2 r$, then $B(x, r) \subset B(0,3 r)$ and we have

$$
\frac{1}{\varphi(r)} \int_{B(x, r)}\left|f_{0}(y)\right|^{p} d y \leq \frac{1}{\varphi(r)} \int_{B(0,3 r)} \frac{\varphi(|y|)}{|y|^{n}} d y .
$$

Passing to polar coordinates and using the growth condition (2.3), we see that

$$
\frac{1}{\varphi(r)} \int_{B(x, r)}\left|f_{0}(y)\right|^{p} d y \lesssim \frac{\varphi(3 r)}{\varphi(r)}
$$

with the implicit constant not depending on $x \in \mathbb{R}^{n}$ and $r>0$. By the assumption (2.4) we see that there exists $c>0$ such that $\frac{\varphi(3 r)}{\varphi(r)} \leq c$ for any $r>0$, and hence

$$
\frac{1}{\varphi(r)} \int_{B(x, r)}\left|f_{0}(y)\right|^{p} d y \leq C<\infty
$$

with $C>0$ independent of $x$ and $r$.

Let now $|x| \geq 2 r$. Since $|x-y| \geq|x|-|y| \geq 2 r-|y| \geq|y|$ for $y \in B(0, r)$, by (2.4) we have

$$
\frac{1}{\varphi(r)} \int_{B(x, r)}\left|f_{0}(y)\right|^{p} d y=\frac{1}{\varphi(r)} \int_{B(0, r)} \frac{\varphi(|x-y|)}{|x-y|^{n}} d y \lesssim \frac{1}{\varphi(r)} \int_{B(0, r)} \frac{\varphi(|y|)}{|y|^{n}} d y .
$$

The desired uniform estimate (with respect to $x$ and $r$ ) follows after passing to polar coordinates and using the assumption (2.3).

Finally, we note that

$$
\int_{\mathbb{R}^{n}}\left|f_{0}(y)\right|^{p} d y \gtrsim \int_{|y| \geq 1}|y|^{-n} d y=\infty
$$

since $\varphi$ is increasing.

Later, in Section 5, we shall consider a further generalization of the Morrey space where one takes a $L^{q}$-(quasi)norm instead of the $L^{\infty}$-norm with respect to $r$ on $(0, \infty)$. For $0<p, q<\infty$, the space $L^{p, q, \varphi}\left(\mathbb{R}^{n}\right)$ is equipped with the (quasi)norm

$$
\|f\|_{L^{p, q, \varphi}}\left(\mathbb{R}^{n}\right):=\sup _{x \in \mathbb{R}^{n}}\left(\int_{0}^{\infty}\left(\frac{1}{\varphi(r)} \int_{B(x, r)}|f(y)|^{p} d y\right)^{q / p} \frac{d r}{r}\right)^{1 / q} .
$$

These spaces were studied, for instance, in [6]. We also refer to the survey paper [15] for further references and historical remarks on this and other generalizations of Morrey spaces that can be found in the literature (see also [19]).

2.2. Generalized Stummel spaces. The classical Stummel space $\mathfrak{S}^{p, \lambda}\left(\mathbb{R}^{n}\right)$ is the space defined by the (quasi)norm

$$
\|f\|_{\mathfrak{S}^{p, \lambda}\left(\mathbb{R}^{n)}\right.}:=\sup _{x \in \mathbb{R}^{n}}\left(\int_{\mathbb{R}^{n}} \frac{|f(y)|^{p}}{|x-y|^{\lambda}} d y\right)^{1 / p}, \quad 0<p<\infty, \quad 0<\lambda<n .
$$

Such a space was used in particular in [9] and [11, 12, 13] in connection to the study of PDE. In a sense, it can be treated as the intersection, with respect to parameter $x_{0} \in \mathbb{R}^{n}$, of weighted Lebesgue spaces with weights $\left|x-x_{0}\right|^{-\lambda}$. There are also known generalizations of such spaces in the spirit of (2.1), i.e., by replacing the power function $r^{\lambda}$ by a more general function $\varphi$ :

$$
\|f\|_{\mathfrak{S}^{p, \varphi}\left(\mathbb{R}^{n}\right)}:=\sup _{x \in \mathbb{R}^{n}}\left(\int_{\mathbb{R}^{n}} \frac{|f(y)|^{p}}{\varphi(|x-y|)} d y\right)^{1 / p} .
$$


We refer, for instance, to the paper [18] where further references can be found.

Following the tradition known for vanishing Morrey spaces and spaces of vanishing mean oscillation (VMO), for $0<p<\infty$ we can also consider the vanishing generalized Stummel class $V_{0} \mathfrak{S}^{p, \varphi}\left(\mathbb{R}^{n}\right)$ as the set of all those functions $f \in \mathfrak{S}^{p, \varphi}\left(\mathbb{R}^{n}\right)$ such that

$$
\lim _{r \rightarrow 0} \sup _{x \in \mathbb{R}^{n}} \int_{B(x, r)} \frac{|f(y)|^{p}}{\varphi(|x-y|)} d y=0 .
$$

For the standard function $\varphi(r)=r^{\lambda}$ we use the natural notation $V_{0} \mathfrak{S}^{p, \lambda}\left(\mathbb{R}^{n}\right)$. Such type of conditions on the function $f$ have been considered in various papers in connection to the study of PDE. For example, they appear in [20] in the case $p=2$; the case $p=1$ is of particular importance (see, for instance, [16]). Note that the case $p=1$ and $\lambda=n-2$ corresponds to the often called Stummel-Kato class.

Examples of functions in $\mathfrak{S}^{p, \lambda}\left(\mathbb{R}^{n}\right)$ and not in $V_{0} \mathfrak{S}^{p, \lambda}\left(\mathbb{R}^{n}\right)$ in one dimensional case can be found in [18, Section 3.2].

We also introduce the following vanishing Stummel class.

Definition 2.2. Let $0<p<\infty$ and $\varphi: \mathbb{R}_{+} \rightarrow \mathbb{R}_{+}$. The class $V_{\infty} \mathfrak{S}^{p, \varphi}\left(\mathbb{R}^{n}\right)$ consists of all functions $f \in \mathfrak{S}^{p, \varphi}\left(\mathbb{R}^{n}\right)$ such that

$$
\lim _{r \rightarrow \infty} \sup _{x \in \mathbb{R}^{n}} \int_{\mathbb{R}^{n} \backslash B(x, r)} \frac{|f(y)|^{p}}{\varphi(|x-y|)} d y=0 .
$$

Some version $V_{\infty} L^{p, \lambda}\left(\mathbb{R}^{n}\right)$ of such a class for Morrey spaces was introduced in [2, Definition 3.1]. It proved to be important for the approximation of functions in Morrey spaces by $C_{0}^{\infty}$ functions.

With respect to the (quasi)norm (2.7), it can be shown that both classes $V_{0} \mathfrak{S}^{p, \varphi}\left(\mathbb{R}^{n}\right)$ and $V_{\infty} \mathfrak{S}^{p, \varphi}\left(\mathbb{R}^{n}\right)$ are closed subspaces of $\mathfrak{S}^{p, \varphi}\left(\mathbb{R}^{n}\right)$.

\section{Embeddings of Generalized Morrey spaCes Between Weighted LEBESGUE SPACES}

In the sequel, for a fixed point $x_{0} \in \mathbb{R}^{n}$, we use the notation

$$
w_{x_{0}}(y):=w\left(\left|y-x_{0}\right|\right) \quad \text { and } \quad \varphi_{x_{0}}(y):=\varphi\left(\left|y-x_{0}\right|\right)
$$

where $w, \varphi$ are positive measurable functions on $\mathbb{R}_{+}$.

In the proof of the main result we need the following auxiliary lemma. The estimates (3.2) and (3.3) below are known and may be found dispersed in various papers. For instance, (3.3) was explicitly formulated in [3, Lemma 3.2].

Lemma 3.1. Let $w: \mathbb{R}_{+} \rightarrow \mathbb{R}_{+}$. If $w(t)$ is almost increasing on $\left(0, R_{1}\right)$, then

$$
\sum_{k=-\infty}^{-1} w\left(2^{k} r\right) \lesssim \int_{0}^{r} \frac{w(t)}{t} d t, \quad 0<r<R_{1} .
$$

If $w(t)$ is almost decreasing on $\left(R_{2}, \infty\right)$, then

$$
\sum_{k=0}^{\infty} w\left(2^{k+1} r\right) \lesssim \int_{r}^{\infty} \frac{w(t)}{t} d t, \quad r>R_{2} .
$$

The main result on embeddings between weighted Lebesgue spaces reads as follows. 
Theorem 3.2. Let $0<p<\infty$ and $x_{0} \in \mathbb{R}^{n}$. Let $\varphi$ be increasing and the weight $w(t)$ be almost increasing on $\left(0, r_{0}\right)$ and almost decreasing on $\left(r_{0}, \infty\right)$ for some $r_{0}>0$, and $\int_{0}^{\infty} \frac{w(t)}{t} d t<\infty$. Then

$$
L^{p}\left(\mathbb{R}^{n}, \frac{1}{\varphi_{x_{0}}}\right) \hookrightarrow L_{\left\{x_{0}\right\}}^{p, \varphi}\left(\mathbb{R}^{n}\right) \hookrightarrow L^{p}\left(\mathbb{R}^{n}, \frac{w_{x_{0}}}{\varphi_{x_{0}}}\right)
$$

and

$$
c\|f\|_{L^{p}\left(\mathbb{R}^{n}, \frac{w_{x_{0}}}{\varphi_{x_{0}}}\right)} \leq\|f\|_{L_{\left\{x_{0}\right\}}^{p, \varphi}\left(\mathbb{R}^{n}\right)} \leq\|f\|_{L^{p}\left(\mathbb{R}^{n}, \frac{1}{\varphi_{x_{0}}}\right)}
$$

where $c=c(p, \varphi, w)$ does not depend on $x_{0}$, if for the right-hand side embedding in (3.4) we additionally assume that $w(t) / \varphi(t)$ is decreasing and $\varphi$ satisfies the doubling condition

$$
\varphi(2 t) \lesssim \varphi(t), \quad t>0 .
$$

Proof. Let $x_{0}=0$ for simplicity. Since $\varphi$ is increasing, the embedding of the left-hand side follows from the inequalities

$$
\int_{\mathbb{R}^{n}}|f(y)|^{p} \frac{1}{\varphi(|y|)} d y \geq \int_{B(0, r)} \frac{|f(y)|^{p}}{\varphi(|y|)} d y \geq \frac{1}{\varphi(r)} \int_{B(0, r)}|f(y)|^{p} d y .
$$

For the other embedding, we use a dyadic decomposition argument as follows. We have

$$
\int_{\mathbb{R}^{n}}|f(y)|^{p} \frac{w(|y|)}{\varphi(|y|)} d y=\sum_{k=-\infty}^{\infty} \int_{2^{k-1} r_{0} \leq|y|<2^{k} r_{0}}|f(y)|^{p} \frac{w(|y|)}{\varphi(|y|)} d y \lesssim \sum_{k=-\infty}^{\infty} w\left(2^{k-1} r_{0}\right)\|f\|_{L_{\{0\}}^{p, \varphi}\left(\mathbb{R}^{n}\right)}^{p}
$$

since $w(t) / \varphi(t)$ is decreasing and $\varphi$ satisfies the doubling condition. Using the estimates (3.2) and (3.3), we get

$$
\int_{\mathbb{R}^{n}}|f(y)|^{p} \frac{w(|y|)}{\varphi(|y|)} d y \lesssim\left(\int_{0}^{r_{0}} \frac{w(t)}{t} d t+w\left(r_{0}\right)+\int_{r_{0}}^{\infty} \frac{w(t)}{t}\right)\|f\|_{L_{\{0\}}^{p, \varphi}\left(\mathbb{R}^{n}\right)}^{p} \lesssim\|f\|_{L_{\{0\}}^{p, \varphi}\left(\mathbb{R}^{n}\right)}^{p},
$$

which completes the proof.

Since the embedding constants in (3.5) do not depend on $x_{0}$, we immediately arrive to the following corollary.

Corollary 3.3. Under the assumptions of Theorem 3.2, for the global Morrey space $L^{p, \varphi}\left(\mathbb{R}^{n}\right)$ there holds the embedding

$$
L^{p, \varphi}\left(\mathbb{R}^{n}\right) \hookrightarrow L^{p}\left(\mathbb{R}^{n}, \frac{w_{x_{0}}}{\varphi_{x_{0}}}\right)
$$

into the weighted space with the weight $\frac{w_{x_{0}}}{\varphi_{x_{0}}}$ fixed at any point $x_{0} \in \mathbb{R}^{n}$.

The result of the corollary generalizes known embeddings obtained in [10] and recently in [2] for classical Morrey spaces, and, for bounded domains, in [18] already for generalized Morrey spaces.

Remark 3.4. An example of a weight satisfying the assumptions of the theorem above is

$$
w(t)=\frac{1}{\ln ^{1+\varepsilon} e \max (t, 1 / t)}, \quad \varepsilon>0 .
$$


Hence Theorem 3.2 recovers similar embeddings from [18] for bounded sets $\Omega \subset \mathbb{R}^{n}$ with

$$
w(t)=\frac{1}{\ln ^{1+\varepsilon} \frac{A}{t}}, \quad A>\operatorname{diam} \Omega
$$

It also recovers an embedding from [2] where locally bounded weights $w$ on $\mathbb{R}^{n}$ were considered.

For weights $w(t)$ satisfying the assumptions of Theorem 3.2, in the next result we use the additional assumption that there exists a positive decreasing function $u(t)$ on $(0,1)$ such that

$$
u(0)=\infty \quad \text { and } \quad \int_{0}^{1} \frac{u(t) w(t)}{t} d t<\infty
$$

The validity of the above condition is obvious for power type weights. For the logarithmic weights in (3.8), which provide a more delicate embedding in (3.7) in comparison with weights $t^{\gamma}$, one can take

$$
u(t)=\ln \ln (e / t) \quad \text { for } 0<t<1 \text {. }
$$

Theorem 3.5. Let $0<p<\infty$ and $x_{0} \in \mathbb{R}^{n}$. Let $\varphi$ be increasing. The left-hand side embedding in (3.4) is strict, i.e., $L^{p}\left(\mathbb{R}^{n}, \frac{1}{\varphi_{x_{0}}}\right) \varsubsetneqq L_{\left\{x_{0}\right\}}^{p, \varphi}\left(\mathbb{R}^{n}\right)$. Let $w(t)$ satisfy the assumptions of Thereom 3.2 and the condition (3.9). Suppose also that $\varphi(t)$ satisfies the assumptions of Lemma 2.1. Then the right-hand side embedding in (3.4) is also strict, i.e., $L_{\left\{x_{0}\right\}}^{p, \varphi}\left(\mathbb{R}^{n}\right) \varsubsetneqq L^{p}\left(\mathbb{R}^{n}, \frac{w_{x_{0}}}{\varphi_{x_{0}}}\right)$.

Proof. For a fixed point $x_{0} \in \mathbb{R}^{n}$ and an increasing function $\varphi$ on $\mathbb{R}_{+}$, we consider the function $f_{0}$ given in $(2.5)$. It is clear that $f_{0} \notin L^{p}\left(\mathbb{R}^{n}, \frac{1}{\varphi_{x_{0}}}\right)$ since

$$
\int_{\mathbb{R}^{n}}\left|f_{0}(y)\right|^{p} \frac{1}{\varphi\left(\left|y-x_{0}\right|\right)} d y=\int_{\mathbb{R}^{n}}\left|y-x_{0}\right|^{-n} d y=\infty .
$$

On the other hand, $f_{0} \in L_{\left\{x_{0}\right\}}^{p, \varphi}\left(\mathbb{R}^{n}\right)$ in view of Lemma 2.1.

Now we show the strictness of the other embedding. Let $g_{0}(x):=\widetilde{u}(|x|)^{\frac{1}{p}} f_{0}(x)$ where $f_{0}$ is the function given in (2.5) and $\widetilde{u}(t)=u(t)$ if $t \in(0,1)$ and $\widetilde{u}(t) \equiv 1$ for $t \geq 1$, with $u(t)$ being the decreasing function on $(0,1)$ satisfying the conditions in $(3.9)$. We have $g_{0} \in L^{p}\left(\mathbb{R}^{n}, \frac{w_{x_{0}}}{\varphi_{x_{0}}}\right)$ since

$$
\begin{aligned}
\int_{\mathbb{R}^{n}}\left|g_{0}(x)\right|^{p} \frac{w\left(\left|x-x_{0}\right|\right)}{\varphi\left(\left|x-x_{0}\right|\right)} d x & =\int_{\left|x-x_{0}\right|<1} \frac{u\left(\left|x-x_{0}\right|\right) w\left(\left|x-x_{0}\right|\right)}{\left|x-x_{0}\right|^{n}} d x+\int_{\left|x-x_{0}\right| \geq 1} \frac{w\left(\left|x-x_{0}\right|\right)}{\left|x-x_{0}\right|^{n}} d x \\
& \approx \int_{0}^{1} \frac{u(t) w(t)}{t} d t+\int_{1}^{\infty} \frac{w(t)}{t} d t<\infty,
\end{aligned}
$$


in view of (3.9) and $\int_{0}^{\infty} \frac{w(t)}{t} d t<\infty$. On the other hand, since $u(t)$ and $\varphi(t) / t^{n}$ decrease, for every $r<1$ we have

$$
\begin{aligned}
\frac{1}{\varphi(r)} \int_{B\left(x_{0}, r\right)}\left|g_{0}(x)\right|^{p} d x & =\frac{1}{\varphi(r)} \int_{B\left(x_{0}, r\right)} \frac{u\left(\left|x-x_{0}\right|\right) \varphi\left(\left|x-x_{0}\right|\right)}{\left|x-x_{0}\right|^{n}} d x \\
& \approx \frac{1}{\varphi(r)} \int_{0}^{r} \frac{u(t) \varphi(t)}{t} d t \\
& \gtrsim \frac{1}{r^{n}} \int_{0}^{r} u(r) t^{n-1} d t=\frac{u(r)}{n} .
\end{aligned}
$$

Therefore, $g_{0} \notin L_{\left\{x_{0}\right\}}^{p, \varphi}\left(\mathbb{R}^{n}\right)$ since $u(r) \rightarrow \infty$ as $r \rightarrow 0$ (by (3.9)).

4. Embeddings of generalized Morrey spaces Between Stummel spaces

From Corollary 3.3 we see that

$$
L^{p, \varphi}\left(\mathbb{R}^{n}\right) \subset \bigcap_{x_{0} \in \mathbb{R}^{n}} L^{p}\left(\mathbb{R}^{n}, \frac{w_{x_{0}}}{\varphi_{x_{0}}}\right)
$$

provided the radial weight $w$ satisfy the assumptions of Theorem 3.2. Moreover, passing to the supremum in (3.5) with respect to $x_{0}$, we conclude that the global generalized Morrey space is continuously embedded between Stummel spaces:

$$
\mathfrak{S}^{p, \varphi}\left(\mathbb{R}^{n}\right) \hookrightarrow L^{p, \varphi}\left(\mathbb{R}^{n}\right) \hookrightarrow \mathfrak{S}^{p, \frac{\varphi}{w}}\left(\mathbb{R}^{n}\right) .
$$

Theorem 4.1. Let $0<p<\infty$. Suppose that $\varphi$ is increasing and satisfies (3.6). Assume also that $w / \varphi$ is almost decreasing. If $w(t)$ is almost increasing on $\left(0, r_{0}\right)$, then

$$
\sup _{x \in \mathbb{R}^{n}} \int_{B(x, r)}|f(y)|^{p} \frac{w(|x-y|)}{\varphi(|x-y|)} d y \lesssim \int_{0}^{r} \frac{w(t)}{t} d t\|f\|_{L^{p, \varphi}\left(\mathbb{R}^{n}\right)}^{p}, \quad 0<r<r_{0} .
$$

If $w(t)$ is almost decreasing on $\left(0, r_{0}\right)$, then

$$
\sup _{x \in \mathbb{R}^{n}} \int_{\mathbb{R}^{n} \backslash B(x, r)}|f(y)|^{p} \frac{w(|x-y|)}{\varphi(|x-y|)} d y \lesssim \int_{r / 2}^{\infty} \frac{w(t)}{t} d t\|f\|_{L^{p, \varphi\left(\mathbb{R}^{n}\right)}}^{p}, \quad r>r_{0} .
$$

Proof. The proof is similar to the second part of the proof of Theorem 3.2. Let $f \in$ $L^{p, \varphi}\left(\mathbb{R}^{n}\right)$. For any $x \in \mathbb{R}^{n}$ and $r>0$, we have

$$
\begin{aligned}
\int_{B(x, r)}|f(y)|^{p} \frac{w(|x-y|)}{\varphi(|x-y|)} d y & =\sum_{k=0}^{\infty} \int_{2^{-k-1} r<|x-y|<2^{-k} r}|f(y)|^{p} \frac{w(|x-y|)}{\varphi(|x-y|)} d y \\
& \lesssim \sum_{k=0}^{\infty} \frac{w\left(2^{-k-1} r\right)}{\varphi\left(2^{-k-1} r\right)} \int_{|x-y|<2^{-k} r}|f(y)|^{p} d y \\
& \lesssim \sum_{k=0}^{\infty} w\left(2^{-k-1} r\right)\|f\|_{L^{p, \varphi\left(\mathbb{R}^{n}\right)}}^{p}
\end{aligned}
$$

Since $w(t)$ increases in $\left(0, r_{0}\right)$, we can estimate the last sum as in (3.2) and we get inequality (4.2). The estimate (4.3) can be derived in a similar way, now using (3.3), observing that $w(t)$ decreases at infinity.

Corollary 4.2. Let $\varphi$ and $w$ satisfy the assumptions of Theorem 4.1. Then

$$
L^{p, \varphi}\left(\mathbb{R}^{n}\right) \hookrightarrow V_{0} \mathfrak{S}^{p, \frac{\varphi}{w}}\left(\mathbb{R}^{n}\right) \cap V_{\infty} \mathfrak{S}^{p, \frac{\varphi}{w}}\left(\mathbb{R}^{n}\right) .
$$


An embedding of the type $L^{p, \varphi}\left(\mathbb{R}^{n}\right) \hookrightarrow V_{0} \mathfrak{S}^{p, \frac{\varphi}{w}}\left(\mathbb{R}^{n}\right)$ was proved in [7] under other assumptions on $\varphi / w$. In that paper the result was formulated as embedding of sets, not as a continuous embedding of spaces.

Finally, in view of the previous results, we arrive at the chain of embeddings

$$
\mathfrak{S}^{p, \varphi}\left(\mathbb{R}^{n}\right) \hookrightarrow L^{p, \varphi}\left(\mathbb{R}^{n}\right) \hookrightarrow V_{0} \mathfrak{S}^{p, \frac{\varphi}{w}}\left(\mathbb{R}^{n}\right) \cap V_{\infty} \mathfrak{S}^{p, \frac{\varphi}{w}}\left(\mathbb{R}^{n}\right) \hookrightarrow \mathfrak{S}^{p, \frac{\varphi}{w}}\left(\mathbb{R}^{n}\right)
$$

provided $\varphi$ and $w$ satisfy the assumptions of last theorem.

\section{On EqUiVAlent (QUASI) NORMS ON StUmmel SPACES}

The coincidence of spaces given in the next theorem was proved in [18] under different assumptions on the function $\varphi$. Here we give a direct proof without using any smoothness condition on $\varphi$, but we have to impose some conditions on the function $\varphi$ near at the origin. We take this opportunity to note that there is a misprint in [18, Corollary 3.4]: the formula $\phi(r)=\frac{r \varphi^{\prime}(r)}{\varphi^{2}(r)}$ there should be $\phi(r)=\frac{\varphi^{2}(r)}{r \varphi^{\prime}(r)}$.

Theorem 5.1. Let $0<p<\infty$. Suppose there exist $\beta>\alpha>0$ such that $\frac{\varphi(t)}{t^{\alpha}}$ is almost increasing and $\frac{\varphi(t)}{t^{\beta}}$ is almost decreasing. Then

$$
\mathfrak{S}^{p, \varphi}\left(\mathbb{R}^{n}\right)=L^{p, p, \varphi}\left(\mathbb{R}^{n}\right)
$$

up to equivalence of (quasi)norms.

Proof. For any $x \in \mathbb{R}^{n}$, we have

$$
\begin{aligned}
\int_{0}^{\infty} \frac{1}{r \varphi(r)} \int_{B(x, r)}|f(y)|^{p} d y d r & =\int_{\mathbb{R}^{n}}|f(y)|^{p}\left(\int_{0}^{\infty} \frac{1}{r \varphi(r)} \chi_{B(x, r)}(y) d r\right) d y \\
& =\int_{\mathbb{R}^{n}}|f(y)|^{p}\left(\int_{|x-y|}^{\infty} \frac{d r}{r \varphi(r)}\right) d y \\
& \approx \int_{\mathbb{R}^{n}} \frac{|f(y)|^{p}}{\varphi(|x-y|)} d y
\end{aligned}
$$

with the implicit constants depending only on $\varphi$ via $\alpha$ and $\beta$. Hence the claim follows after passing to the supremum on $x \in \mathbb{R}^{n}$.

\section{REFERENCES}

[1] D.R. Adams: Morrey Spaces, Lecture Notes in Applied and Numerical Harmonic Analysis, Birkhäuser, 2015.

[2] A. Almeida, S. Samko: Approximation in Morrey spaces, J. Funct. Anal. 272 (2017), 2392-2411.

[3] E. Burtseva, N. Samko: Weighted Adams type theorem for the Riesz fractional integral in generalized Morrey spaces, Fract. Calc. Appl. Anal. 19 (2016), 954-972.

[4] G. Citti, G. Di Fazio: Hölder continuity of the solutions for operators which are a sum of squares of vector fields plus a potential, Proc. Amer. Math. Soc. 122 no. 3 (1994), 741-750.

[5] M. Giaquinta: Multiple integrals in the calculus of variations and non-linear elliptic systems, Princeton Univ. Press, Princeton, 1983.

[6] V. Guliyev: Function spaces, integral operators and two weighted inequalities on homogeneous groups. Some applications (in Russian), Casioglu, Baku, 1999.

[7] H. Gunawan, E. Nakai, Y. Sawano, H. Tanaka: Generalized Stummel class and Morrey spaces, Publications de L'Institut Mathématique, Nouvelle série, tome 92(106) (2012), 127-138.

[8] D. Haroske, L. Skrzypczak: Embeddings of weighted Morrey spaces, Math. Nachr. 290 (2017), no. 7, 1066-1086. 
[9] A.I. Koshelev: Regularity problem for quasilinear elliptic and parabolic systems, Springer, Berlin, 1995.

[10] T. Kato: Strong solutions of the Navier-Stokes equation in Morrey spaces, Bol. Soc. Bras. Mat. 22 (1992), 127-155.

[11] S. Leonardi: Remarks on the regularity of solutions of elliptic systems, A. Sequeira, H.B. Veiga, J.H. Videman (Eds.), Applied Nonlinear Analysis, Kluwer, New York, 1999, 325-344.

[12] S. Leonardi: Weighted Miranda-Talenti inequality and applications to equations with discontinuous coefficients, Comment. Math. Univ. Carolin. 43 no. 1 (2002), 43-59.

[13] S. Leonardi, J. Kottas, J. Stará: Hölder regularity of the solutions of some class of elliptic systems in convex nonsmooth domains, Nonlinear Anal. 60 (2005), 925-944.

[14] L. Pick, A. Kufner, O. John, S. Fučík: Function spaces, Vol. 1, 2nd. ed., De Gruyter Series in Nonlinear Analysis and Applications 14, Berlin, 2013.

[15] H. Rafeiro, N. Samko, S. Samko: Morrey-Campanato spaces: an overview, In: Karlovich, Yi., Rodino, L., Silbermann, B., Spitkovsky, IM. (eds.) Operator Theory, Pseudo-Differential Equations and Mathematical Physics, Advances and Applications, vol. 228, pp. 293-323, Springer, Basel, 2013.

[16] M.A. Ragusa, P. Zamboni: A potential theoretic inequality, Czech. Math. J. 51(126) (2001), 55-65.

[17] M. Rosenthal, H. Triebel: Morrey spaces, their duals and preduals, Rev. Mat. Complut. 28 (2015), $1-30$.

[18] S. Samko: Morrey spaces are closely embedded between vanishing Stummel spaces, Math. Ineq. Appl. 17, no. 2 (2014), 627-639.

[19] Y. Sawano, H. Gunawan, V. Guliyev, H. Tanaka: Morrey spaces and related function spaces, J. Funct. Spaces, Vol. 2014, Article ID867192.

[20] F. Stummel: Singuläre elliptische Differential-operatorenin Hilbertschen Räumen, Math. Ann. 132 (1956), 150-176.

[21] M.E. Taylor: Tools for PDE: Pseudodifferential Operators, Paradifferential Operators and Layer Potentials, Math. Surveys and Monogr., Vol. 81, AMS, Providence, 2000.

[22] H. Triebel: Local function spaces, Heat and Navier-Stokes equations, EMS Tracts in Mathematics $20,2013$.

[23] H. Triebel: Hybrid function spaces, Heat and Navier-Stokes equations, EMS Tracts in Mathematics $24,2015$.

[24] H. Wadade: Optimal embeddings of critical Sobolev-Lorentz-Zygmund spaces Stud. Math. 223, no.1 (2014), 77-95.

[25] P. Zamboni: Some function spaces and elliptic partial differential equations, Le Matematiche, Vol. XLII, Fasc. I-II (1987), 171-178.

Department of Mathematics, Center for R\&D in Mathematics and Applications, UniVersity of Aveiro, 3810-193 Aveiro, Portugal

E-mail address: jaralmeida@ua.pt

University of Algarve, Department of Mathematics, Campus de Gambelas, 8005-139 FAro, Portugal

E-mail address: ssamko@ualg.pt 